\title{
Moments of Inertia of SDIBs in $A=190$ Mass Region using VMI Model
}

\author{
P. Jain ${ }^{1 *}$, A. Goel ${ }^{1}$, S. K. Mandal ${ }^{2}$ \\ ${ }^{1}$ Amity Institute of Nuclear Science and Technology, Amity University, Uttar Pradesh-201303, India \\ ${ }^{2}$ Department of Physics and Astrophysics, University of Delhi, Delhi-110007, India
}

Received 20 September 2019, accepted in final revised form 26 December 2019

\begin{abstract}
A lot of identical bands are known at present in the Normal Deformed (ND) region. In our study of the occurrence and properties of identical bands in Super-Deformed (SD) nuclei we first applied the modified Variable Moment of Inertia (VMI) model to extract the band-head spin of Super-Deformed bands. The calculated transition energies, level spins and dynamic moment of inertia are systematically examined. Then, in the framework of theoretical model several identical bands are identified. The kinematic and dynamic moment of inertia have been calculated for the six pairs of Super-Deformed Identical Bands (SDIBs) which was not reported earlier in the literature. Thus, the results are significant. In all the cases $J^{(2)}$ is significantly higher than $J^{(1)}$ over a large range of frequency.
\end{abstract}

Keywords: Super-Deformed band; Identical band; VMI; BFM.

(C) 2020 JSR Publications. ISSN: 2070-0237 (Print); 2070-0245 (Online). All rights reserved. doi: http://dx.doi.org/10.3329/jsr.v12i2.43867

J. Sci. Res. 12 (2), 209-214 (2020)

\section{Introduction}

The discovery of Identical Bands (IBs) in both Super-Deformed and Normal Deformed bands surprised and initialized many new experimental as well as theoretical studies [1, 2]. Observation of rotational bands in two different nuclei which have nearly same transition energies $E$ within $\pm 3 k e V$ and nearly identical moment of inertia (dynamic) called identical bands was long believed would be difficult in nuclear physics. Therefore, finding such rotational bands increased the considerable interest in the field of nuclear physics. These identical bands exist at both low and high spins and thus describe many features of Super-Deformed and Normal Deformed bands. The first pair of SD identical bands was an excited band in ${ }^{151} \mathrm{~Tb}$ whose energies were identical to yrast band of ${ }^{152} \mathrm{Dy}$ [3]. Later six identical SD bands found in ${ }^{194} \mathrm{Tl}$ [4]. In the past years, there have been a lot of theoretical works presented on various nuclear models to observe IBs both in ND and SD nuclei [5-12]. The above study motivated us to study the various features of SDIBs in $A=190$ mass region. So the purpose of this work is to point out the existence of IBs in the

*Corresponding author: poonam.jn1@gmail.com 
SD nuclei with the help of modified VMI model and examine the behaviours of kinematic and dynamic moments of inertia.

The paper is organised as follows: A brief description of our approach to predict the level spin and band-head spin along with the calculation for kinematic and dynamic moments of inertia are presented in section 2. In Section 3 we present the obtained results for IBs in $A=190$ mass region for SD nuclei. Finally, conclusion is given in section 4 .

\section{A Brief description of the VMI Model}

The complete explanation of VMI model is described in literature [13-15]. The energy level of $I_{0}=0$, the ground-state bands in even-even nuclei are represented as:

$E_{I}(J)=\frac{1}{2} C\left(J_{I}-J_{0}\right)^{2}+\frac{1}{2}\left[\frac{I(I+1)}{J_{I}}\right]$

The cubic equation is taken from the model. This cubic equation has one real root for any finite positive value of $J_{0}$ and $C$. The cubic equation is given as:

$J_{I}^{3}-J_{I}^{2} J_{0}-([I(I+1)] / 2 C)=0$

The transition energy for SD band is defined as [16]:

$$
E_{\gamma}(I \rightarrow I-2)=\frac{1}{2 J_{0}}[I(I+1)-(I-2)(I-1)]+\frac{1}{8 C J_{0}^{4}}\left([I(I+1)]^{2}-[(I-2)(I-1)]^{2}\right)
$$

In this equation, the parameters $J_{0}$ and $C$ are determined by fitting the experimentally known transition energies by using the Best-Fit Method (BFM). To find the rotational frequency $\hbar \omega$, the kinematic $J^{(1)}$ and dynamic $J^{(2)}$ moment of inertia using literature [1315], we have written an equation by differentiating the energy with respect to $I(I+1)$ and using the chain rule, we can extract $\hbar \omega ; J^{(1)}$ and $J^{(2)}$ from their definitions:

$$
\begin{aligned}
& \hbar \omega=\frac{d E}{d[I(I+1)]}, \\
& J^{(1)}=\hbar^{2}[I(I+1)]^{1 / 2}\left(\frac{d E}{d[I(I+1)]}\right)^{-1} \approx \frac{2 I-1}{E_{\gamma}(I \rightarrow I-2)} \\
& J^{(2)}=\hbar^{2}\left(\frac{d^{2} E}{d[I(I+1)]^{2}}\right)^{-1} \approx \frac{4}{E_{\gamma}(I+2 \rightarrow I)-E_{\gamma}(I \rightarrow I-2)}
\end{aligned}
$$

The $J^{(1)}$ moment of inertia is a direct measure of the transition energies while $J^{(2)}$ is obtained from differences in transitions energies (relative change in transition energies). The $J^{(I)}$ kinematic moment of inertia and $J^{(2)}$ dynamic moment of inertia are plotted with $(ち \omega)$ rotational frequency to obtain the identical bands for SD nuclei. 
Table 1. Band head moment of inertia, experimental transition energies, calculated transition energies, rotational frequencies along with kinematic $J^{(1)}$ and dynamic moments of inertia $J^{(2)}$ for SDIBs in $A=190$ mass region are given. The first row shows the band head moment of inertia $\left(J_{0}\right)$, the second row gives the estimated spin, the third and fourth rows gives the experimental and calculated transition energies respectively. In the fifth row the rotational frequencies are given. The kinematic and dynamic moment of inertia are presented in the sixth and seventh row respectively.

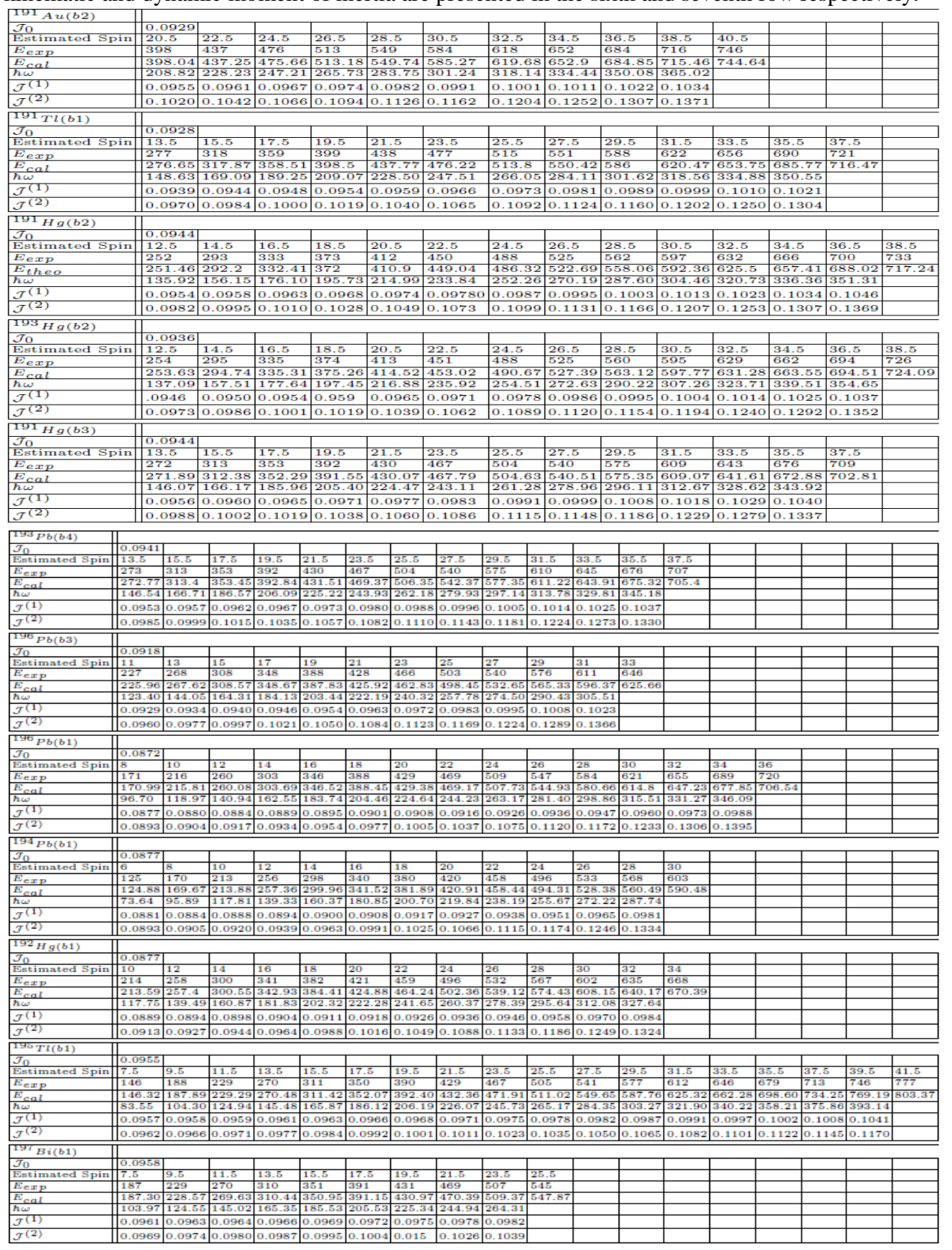




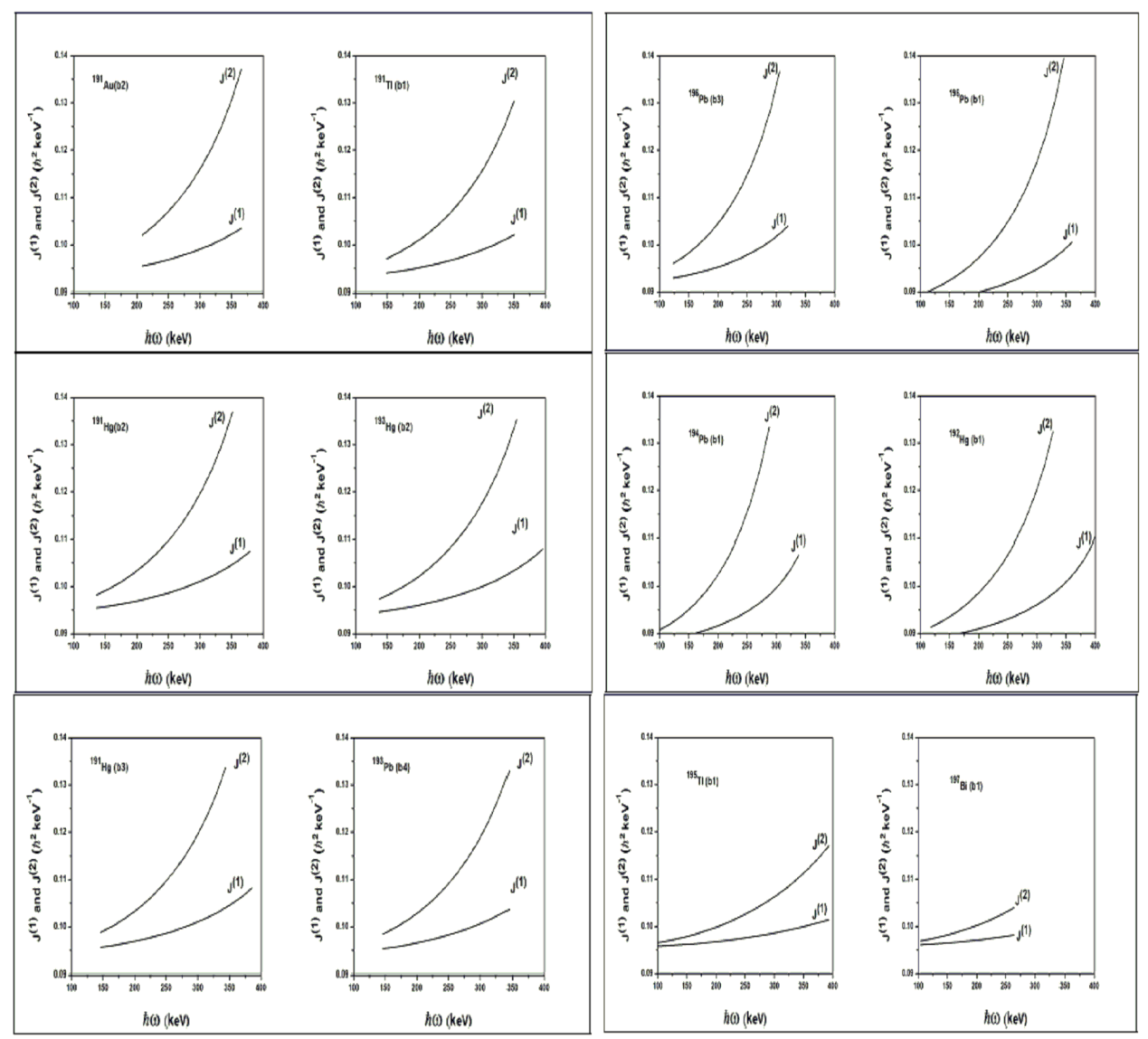

Fig. 1. The kinematic $J^{(1)}$ and dynamic $J^{(2)}$ moment of inertia plots with rotational frequency for 6 pairs of SDIBs. $J^{(2)}$ is significantly higher than $J^{(1)}$ in all the cases.

\section{Results}

In this section we studied 16 pairs of Super-Deformed Identical Bands (SDIBs) in $A=190$ mass region. Firstly the band head spin and level spins of all the available SD bands is obtained by using the modified VMI model. The transition energies, band head moment of inertia for all the pairs have been calculated and compared with the experimentally available data using modified VMI model and data for 6 pairs are presented in Table 1 which are not quoted earlier in literature. The band-head moment of inertia $\left(J_{0}\right)$ parameter is determined by fitting experimentally known transition energies using the Best Fit Method (BFM). A good agreement between the moment of inertia and the energies between two isotopes confirm these as identical bands which are investigated and shown in Table 1. The band-head moment of inertia $\left(J_{0}\right)$, calculated and experimental transition energies, rotational frequencies, kinematic $J^{(1)}$ and dynamic $J^{(2)}$ moment of inertia along with spin for the six pairs of IBs are given in Table 1. To illustrate the quantitative 
agreement between the two isotopes of identical bands, we plotted in Fig. 1 the kinematic $J^{(1)}$ and dynamic $J^{(2)}$ moments of inertia with rotational frequencies for 6 pairs of IBs in which each pair has same behaviour. The results are in good agreement with each other. The $J^{(2)}$ is significantly higher than $J^{(1)}$ over a large rotational frequency range in $A=190$ mass region.

\section{Conclusion}

The present study is an effort to explore the SDIBs in $A=190$ mass region. We have been able to confirm 16 pairs of IBs firmly in 190 mass region. It is well known that the two rotational bands having same dynamic and kinematic moment of inertia are considered to be as identical to each other. This fact seems to suggest that the band-head moment of inertia of these bands should be same. The problem of identical bands in Super-Deformed nuclei is investigated successfully. Six pairs of IBs are reported which was not mentioned earlier in the literature. With the help of modified VMI model, we find an excellent agreement between the experimental and theoretical energies. Also, we calculated kinematic and dynamic moment of inertia along with rotational frequencies and in all the six cases the results are in good agreement within the pair. In all the cases $J^{(2)}$ is significantly higher than $J^{(1)}$. This work can be helpful in the field of nuclear physics.

\section{References:}

1. C. Baktash, B. Haas, and W. Nazarewicz, Annu. Rev. Nucl. Part. Sci. 45, 485 (1995). https://doi.org/10.1146/annurev.ns.45.120195.002413

2. L. B. Karlsson, I. Ragnarsson, and S. Aberg, Phys. Lett. B 416, 16 (1998). https://doi.org/10.1016/S0370-2693(97)01324-5

3. T. Byrski, F. A. Beck, D. Curien, C. Schuck, P. Fallon, A. Alderson, I. Ali, M. A. Bentley, A. M. Bruce, P. D. Forsyth, D. Howe, J. W. Roberts, J. F. Sharpey-Schafer, G. Smith, and P. J. Twin, Phys. Rev. Lett. 64, 1650 (1990). https://doi.org/10.1103/PhysRevLett.64.1650

4. F. Azaiez, W. H. Kelly, W. Korten, F. S. Stephens, M. A. Deleplanque, R. M. Diamond, A. O. Macchiavelli, J. E. Draper, E. C. Rubel, C. W. Beausang, J. Burde, J. A. Becker, E. A. Henry, S. W. Yates, M. J. Brinkman, A. Kuhnert, and T. F. Wang, Phys. Rev. Lett. 66, 1030 (1991).

5. I. Ahmad, M. P. Carpenter, R. R. Chasman, R. V. F. Janssens, and T. L. Khoo, Phys. Rev. C 44, 1204 (1991). https://doi.org/10.1103/PhysRevC.44.1204

6. R. F. Casten, N. V. Zamr, P. B. Von, and W. T. Chou, Phys. Rev. C 45, R1413 (1992). https://doi.org/10.1103/PhysRevC.45.R1413

7. M. Saha and S. Sen, Phys. Rev. C 46, R1587 (1992). https://doi.org/10.1103/PhysRevC.46.R1587

8. A. M. Khalaf, M. D. Okasha, and K. M. Abdelbased, Prog. Phys. 13, 50 (2017).

9. C. Baktash, J. D. Garrett, Phys. Rev. Lett. 69, 1500 (1992). https://doi.org/10.1103/PhysRevLett.69.1500

10. J. Y. Zeng, S. X. Liu, Y. A. Lei, and L. Yu, Phys. Rev. C 63, 024305 (2001).

11. C. Baktash, D. F. Winchell, J. D. Garrett, and A. Smith, Nucl. Phys. A 557, 145 (1993). https://doi.org/10.1016/0375-9474(93)90537-8

12. M. Saha and S. Sen, Phys. Rev. C 50, 2794 (1994). https://doi.org/10.1103/PhysRevC.50.2794

13. M. A. J. Mariscotti, G. Scharff-Goldhaber, and B. Buck, Phys. Rev. 178, 1864 (1969). https://doi.org/10.1103/PhysRev.178.1864 
14. A. K. Jain and A. Goel, Int. J. Mod. Phys. E 02, 451 (1993). https://doi.org/10.1142/S0218301393000170

15. P. Jain, V. S. Uma, A. Goel, and S. K. Mandal, Eur. Phys. J. Plus 134, 72 (2019). https://doi.org/10.1140/epjp/i2019-12443-3

16. V. S. Uma, A. Goel, A. Yadav, and A. K. Jain, Pram. J. Phys. 86, 185 (2016). https://doi.org/10.1007/s12043-015-1013-9 